\title{
CALIBRAÇÃO DO MODELO ORYZA-APSIM PARA O ARROZ DE TERRAS ALTAS NO BRASIL
}

\author{
Rogério Lorençoni ${ }^{1}$, Durval Dourado Neto $^{1}$, Alexandre Bryan Heinemann², Leonardo Cirilo da \\ Silva Soares ${ }^{1}$, Adilson Nunes da Siva ${ }^{1}$
}

' Departamento de Produção Vegetal - ESALQ/USP, CEP : 13.418-900, Piracicaba, SP. E-mail: dourado@esalq.usp.br
${ }^{2}$ EMBRAPA Arroz e Feijão, Caixa Postal 179, 75375-000, Santo Antônio de Goiás, GO. E-mail: alexbh@cnpaf.embrapa.br

\section{RESUMO}

Com o objetivo de calibrar o modelo ORYZA-APSIM para o arroz de terras altas no Brasil, foram conduzidos dezessete experimentos pela EMBRAPA Arroz e Feijão, em quatro regiões brasileiras (Santo Antônio de Goiás-GO, Sorriso-MT, Gurupi-TO e Teresina-PI) produtoras de arroz de terras altas, entre as safras de 2005/2006 e 2008/2009. O modelo foi calibrado à variedade cultivada de arroz de terras altas BRS-Primavera. As seguintes variáveis foram definidas na calibração: (i) unidades de calor efetivo diário, (ii) taxas de desenvolvimento fenológico para cada estágio, (iii) frações de massa de matéria seca das folhas, colmos e órgãos de armazenagem durante diferentes fases de desenvolvimento da cultura, (iv) taxa de crescimento inicial da área foliar e (v) parâmetros utilizados para determinar a área foliar específica. $\mathrm{Na}$ avaliação do modelo, foram comparados valores simuladas com valores observados da duração (número de dias) do período entre a emergência e o florescimento da cultura de arroz de terras altas. O modelo não apresentou desempenho satisfatório na simulação da duração das diferentes fases fenológicas de desenvolvimento para a região de Teresina-PI (os números de dias simulados foram, em média, oito dias superiores aos valores observados).

Palavras-chave: Oryza sativa, BRS-Primavera, Modelagem

\section{CALIBRATION OF THE ORYZA-APSIM MODEL FOR THE UPLAND RICE IN BRAZIL}

\begin{abstract}
With the purpose of calibrating and evaluating the ORYZA-APSIM model for the upland rice in Brazil, 17 field experiments were carried out at EMBRAPA, in four upland rice Brazilian regions (Santo Antônio de Goiás-GO, Sorriso-MT, Gurupi-TO and Teresina-PI), from 2005/2006 to 2008/2009 seasons. The model was calibrated and evaluated for an upland rice, cultivar BRSPrimavera. The following variables were defined in the calibration: (i) daily effective heat units, (ii) phenological development rates at each crop stage, (iii) fractions of the dry matter of leaves, stems and storage organs during the developmental crop stages, (iv) initial growth rate of leaf area and (v) parameters used to define the specific leaf area. The model did not present satisfactory performance to simulate the duration of different phenological phases of development for the Teresina-PI region (the numbers of simulated days were, in average, eight days higher than the observed values).
\end{abstract}

Key words: Oryza sativa, BRS-Primavera, Modeling 


\section{INTRODUÇÃO}

A otimização dos recursos naturais, aliada as práticas de manejo adaptadas a diferentes ambientes inseridos na área de produção de arroz de terras altas, pode contribuir para minimizar a variabilidade temporal e espacial da produtividade. Modelos de simulação do crescimento e desenvolvimento de culturas são ferramentas que foram desenvolvidas para múltiplas aplicações na pesquisa agropecuária (Hoogenboom et al., 1999; Jones et al., 2003). Eles podem ser utilizadas para integrar os conhecimentos dos processos biofísicas que regem o sistema solo-águaplanta, permitindo identificar e avaliar as incertezas na produção, associadas às diferentes opções de manejo. Os modelos também tornam possível aperfeiçoar a eficiência da pesquisa, permitindo a análise do desempenho de cultivares em diferentes solos, condições climáticas, datas de semeadura, densidades de plantas, manejo da irrigação e épocas de aplicação de nitrogênio (Heinemann et al. 2000; Heinemann et al., 2002; Zhang et al., 2007. Um desses modelos é o ORYZA 2000, que foi desenvolvido pela Universidade de Wageningen e o Instituto Internacional de Pesquisa em Arroz (IRRI) nos anos 90. Esse modelo simula a dinâmica do crescimento e desenvolvimento da cultura do arroz para condições ótimas, que levam ao rendimento potencial (Kropff et al., 1994), para condições limitantes de nitrogênio ou para a otimização da aplicação de nitrogênio, assumindo-se que, em todas estas situações a cultura está bem protegida e não sofre reduções de produtividade devido a doenças, pragas e ervas daninhas (Bouman \& Laar, 2006). Recentemente, o modelo ORYZA 2000 foi incorporado ao sistema de suporte a decisão "Agricultural Production Systems Simulator" (APSIM) e vem sendo utilizado para embasar decisões agronômicas e alocação de recursos na Australia para a cultura do arroz, como também para rotações de culturas (Gaydon et al., 2006). ). A incorporação do ORYZA2000 ao sistema de suporte de decisão APSIM foi designada para a simulação de sistemas agrícolas complexos e de seus respectivos manejos, possibilitando o uso do modelo para o sistema de cultivo de arroz de terras altas. $\mathrm{O}$ modelo ORYZA-APSIM permite a realização de estudos sobre a sustentabilidade do sistema de produção de arroz, simulando a comportamento da cultura sob diferentes condições de solos, climas e manejo. Um modelo simulação, quando bem calibrado, pode, dentre outras coisas, determinar tendências da produtividade de genótipos sob diferentes condições ambientais. O modelo de simulação ORYZA-APSIM pode ser calibrado para caracterizar o desenvolvimento, crescimento e a ordem de grandeza da produtividade $\mathrm{da}$ variedade cultivada de terras altas BRS-Primavera com base nos dados de solo, clima e genótipo (características do ambiente de diferentes regiões produtoras de arroz de terras altas.

O objetivo deste trabalho foi calibrar o modelo ORYZA-APSIM em simular o desenvolvimento da variedade cultivada de arroz de terras altas BRS-Primavera nas condições ambientais da calibração e em quatro diferentes regiões produtoras de arroz no Brasil.

\section{MATERIAIS E MÉTODOS}

Os dados da calibração foram obtidos de dezessete experimentos pela EMBRAPA Arroz e Feijão, em quatro regiões brasileiras (Santo Antônio de Goiás-GO, Sorriso-MT, Gurupi-TO e Teresina-PI) produtoras de arroz de terras altas, entre as safras de $2005 / 2006$ e 2008/2009. Foram realizadas calibrações para a determinação do desenvolvimento fenológico (duração das fases 1 e 2) nos dois experimentos nas duas 
datas de plantio. A sensibilidade fotoperiódica do genótipo foi avaliada para se determinar o comportamento da cultura durante a fase vegetativa. $\mathrm{Na}$ avaliação da sensibilidade fotoperiódica, foi realizada uma regressão linear do número de dias observados na fase vegetativa em função das respectivas datas de semeadura, onde o valor do coeficiente angular da regressão foi utilizado para determinar a sensibilidade da variedade cultivada (Figura $2 \mathrm{~B}$ ). O valor do coeficiente angular determinado para a variedade cultivada BRS-Primavera foi comparado com valores estabelecidos por Fukai (1999). Foram determinadas as unidades de calor efetivo diário $H U\left({ }^{\circ} \mathrm{Cd}^{-1}\right) \mathrm{e}$ as taxas de desenvolvimento de cada um dos seus respectivos estádios fenológicos $(D V R)$. As unidades de calor efetivo $(H U H)$ foram determinadas em função das temperaturas cardinais fixadas pelo modelo $\left(T_{b}=8^{\circ} \mathrm{C} ; T_{O}=30^{\circ} \mathrm{C} ; T_{M}=42^{\circ} \mathrm{C}\right)$ e pela temperatura horária, obtida pela equação (1).

$$
T_{d}=\frac{T_{m}+T_{M}}{2}+\frac{T_{M}-T_{m}}{2} \cos [0,2618 h-14]
$$

em que $h$ é o período do dia.

$$
\begin{gathered}
H U H=0\left(\text { se } \mathrm{T}_{\mathrm{d}} \leq \mathrm{T}_{\mathrm{b}} \text { ou } \mathrm{T}_{\mathrm{d}} \geq T_{M}\right) \\
H U H=\frac{T_{d}-T_{b}}{24}\left(\text { se } \mathrm{T}_{\mathrm{b}}<\mathrm{T}_{\mathrm{d}}<\mathrm{T}_{\mathrm{M}}\right) \\
H U H=\frac{1}{24}\left[T_{o}-T_{d}-T_{O} \frac{T_{O}-T_{b}}{T_{H}-T_{O}}-T_{b}\right]\left(\text { se } \mathrm{T}_{\mathrm{O}}<\mathrm{T}_{\mathrm{d}}<\mathrm{T}_{\mathrm{M}}\right)
\end{gathered}
$$

O DVR foi determinado de acordo com Kropff et al. (1994), baseado nas taxas constantes dos estádios fenológicos e suas unidades de calor efetivas diário ( $H U$;

$\left.{ }^{\circ} \mathrm{C} . d i a . d i a^{-1}\right)$ ocorridas no estádio. $H U\left({ }^{\circ} \mathrm{Cd} \mathrm{d}^{-1}\right)$ foi obtido pela seguinte equação:

$$
H U=\sum_{h=1}^{24} H U H_{h}
$$

Então o $D V R$ e o $D V S$ são obtidos pelas seguintes equações:

$$
D V R=\frac{D V S}{H U}(\text { ou } D V S=D V R \cdot H U)
$$


Para minimizar o efeito de possíveis erros experimentais, foram utilizados os valores médios dos dados observados nos dois experimentos.

\section{Partição de assimilados}

A partição de assimilados foi determinada em função da fração da massa de matéria seca dos órgãos contidos na biomassa seca total da parte aérea observada nos experimentos. As frações da massa de matéria seca dos órgãos na biomassa seca total da parte aérea foram assim calculadas:

$$
\begin{aligned}
& F L V=\frac{\Psi_{t_{2}}-\Psi_{t_{1}}}{\Omega_{t_{2}}-\Omega_{t_{1}}} \\
& F S T=\frac{\Upsilon_{t_{2}}-\Upsilon_{t_{1}}}{\Omega_{t_{2}}-\Omega_{t_{1}}} \\
& F S O=\frac{\chi_{t_{2}}-\chi_{t_{1}}}{\Omega_{t_{2}}-\Omega_{t_{1}}}
\end{aligned}
$$

em que $F L V, F S T$ e $F S O$ se referem, respectivamente, às frações $\left(\mathrm{kg}_{\mathrm{kg}} \mathrm{kg}^{-1}\right) \mathrm{da}$ matéria seca da parte aérea alocada para folhas, colmos e órgãos de armazenagem; $\Psi, \chi$ e $\Omega$ se referem, respectivamente, à massa $(\mathrm{kg})$ da folha, da panícula e da parte aérea nos instantes $t_{1}$ e $t_{2}$ (instantes iniciais e finais referentes ao início e fim do período de avaliação).

A fração da massa de matéria seca de colmos de reserva (FSTR, $\mathrm{kg}^{\mathrm{kg}} \mathrm{kg}^{-1}$ ) é assim calculada:

$$
F S T R=\frac{\beta-\delta}{\beta}
$$

em que $\beta$ se refere à massa máxima do colmo $(\mathrm{kg})$ no florescimento e $\delta$ à massa do colmo (kg) na colheita.

As curvas de partição de assimilados utilizadas na calibração do modelo foram determinadas e função das frações da biomassa seca dos órgãos e biomassa seca total da parte aérea observadas nos diferentes estágios de desenvolvimento da cultura. Frações de matéria seca dos órgãos em estágios de desenvolvimento intermediários aos observados nos experimentos foram utilizadas na construção das curvas. Essas frações foram obtidas por interpolação dos valores observados em diferentes estágios de desenvolvimento da cultura. Comparações entre a curva padrão do genótipo de arroz irrigado (variedade cultivada IR-72) e a curva determinada para a variedade cultivada de arroz de terras altas BRSPrimavera são apresentadas na Figura 1. 

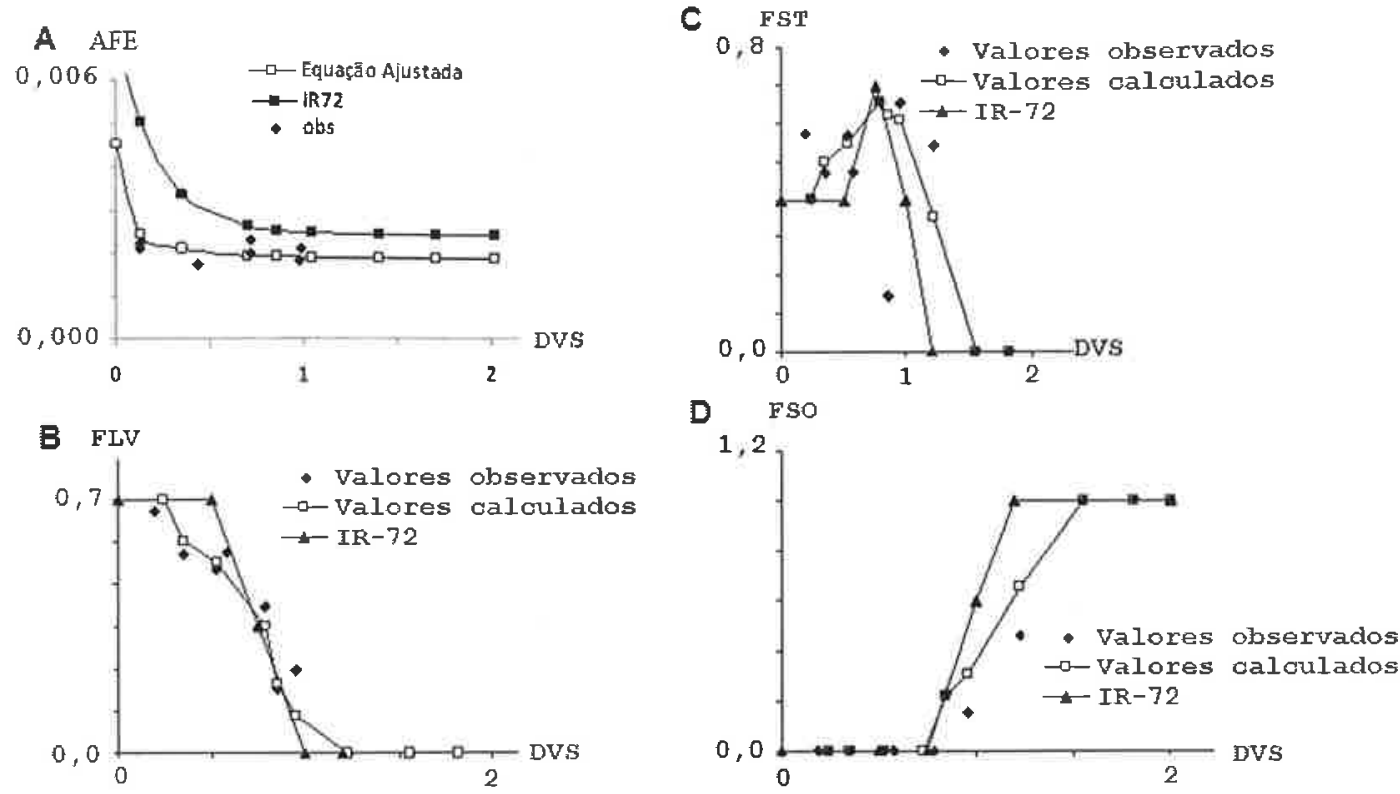

Figura 1. (A) curva da área foliar específica $\left(A F E\right.$, ha. $\mathrm{kg}^{-1}$ - ha de folha por $\mathrm{kg}$ de folha) ajustada pelos parâmetros a e b em função dos valores observados; Curva de partição de assimilados da variedade cultivada irrigada IR-72 e da variedade cultivada de terras altas BRS-Primavera: (B) para as folhas $\left(F L V, \mathrm{~kg} . \mathrm{kg}^{-1}\right)$; (C) para os colmos $(F S T$, kg.kg-1); (D) para os órgãos de armazenagem (FSO, kg. $\left.\mathrm{kg}^{-1}\right)$

\section{Índice de área foliar}

O crescimento foliar é dividido em duas fases. A primeira fase do crescimento foliar, quando (Índice de área foliar igual ou inferior a 1), é caracterizada pelo crescimento exponencial do $I A F$ em função da soma térmica ocorrida no período. A segunda fase do crescimento foliar, quando (Índice de área foliar superior a 1), é caracterizada pelo crescimento linear do IAF em função da área foliar específica $(A F E$, ha. $\mathrm{kg}^{-1}$ ).

$\mathrm{Na}$ fase inicial do crescimento foliar (exponencial), o $I A F$ foi calculado em função da soma térmica e da taxa de crescimento relativo da área foliar $\left(R G R L,\left({ }^{\circ} \mathrm{C} \cdot \text { dia }\right)^{-1}\right)$. O cálculo do $I A F$ é efetuado utilizando a seguinte equação:

$$
I A F_{t_{s}}=I A F_{t_{0}} \cdot e^{R G R L . t_{s}}
$$

em que $I A F$, se refere ao índice de área foliar $\left(\mathrm{m}^{2} \cdot \mathrm{m}^{-2}\right)$ em uma soma de temperatura específica após a emergência $t_{s}\left({ }^{\circ} \mathrm{C}\right.$.dia $)$ e $I A F_{t_{0}}$ ao índice de área foliar $\left(\mathrm{m}^{2} \cdot \mathrm{m}^{-2}\right)$ na soma de temperatura zero $\left(t_{0},{ }^{\circ} \mathrm{C}\right.$.dia $)$. A RGRL é estimada a partir da inclinação da relação entre $\ln (I A F)$ e a soma das unidades de calor efetivo diário ( $H U$, ${ }^{\circ} \mathrm{C}$. dia): 


$$
R G R L=\frac{\ln \left(I A F_{i_{2}}\right)-\ln \left(I A F_{t_{1}}\right)}{H U}
$$

em que $I A F_{t_{2}}$ é o índice de área foliar na soma de temperatura final da fase exponencial e $I A F_{t_{1}}$ é o índice de área foliar na soma da temperatura no inicio da fase exponencial.

$\mathrm{O}$ rápido crescimento da área foliar do genótipo de terras altas durante a fase exponencial, impossibilitou a coleta de dados do $I A F$ nessa fase. Devido à falta de dados, o valor da $R G R L$ foi obtido por meio de simulações onde foram testados diferentes valores para a $R G R L$.

$\mathrm{Na}$ segunda fase do crescimento foliar (linear), a taxa de crescimento da área foliar foi obtida em função da área foliar específica. A área foliar específica $(A F E$, ha folha por $\mathrm{kg}$ de folha) foi obtida pela equação:

$$
A F E=a-b e^{c(D V S-d)}
$$

em que $a, b, c$ e $d$ são parâmetros da função. Esses parâmetros determinam o formato e o patamar da curva.

Para a calibração do $I A F$ no modelo, foram determinados valores para os parâmetros $a \mathrm{e}$ $b$. Já para os parâmetros $c$ e $d$, foram utilizados os valores padrão estabelecidos no modelo. Os valores dos parâmetros $a$ e $b$, foram determinados por meio de simulações, onde foram utilizados diferentes valores para esses parâmetros (tentativa e erro), até se obter um ajuste aproximado da curva do $A F E$ com os valores observados. A curva ajustada para a calibração do modelo é apresentada na Figura 2.

Os valores dos parâmetros empíricos referentes à estimativa da área foliar específica, da fração da massa de matéria seca de colmos de reserva utilizados na calibração do modelo e da taxa de crescimento relativo da área foliar são demonstrados na Tabela 1.

Tabela 1. Parâmetros $(a, b, c$ e $d)$ empíricos referentes à estimativa da área foliar específica, fração da massa de matéria seca de colmos de reserva (FSTR, $\left.\mathrm{kg}_{\mathrm{kg}} \mathrm{kg}^{-1}\right)$ e taxa de

\begin{tabular}{|c|c|c|c|c|c|c|}
\hline $\begin{array}{l}\text { Dados da } \\
\text { calibração }\end{array}$ & $\begin{array}{c}a \\
\text { ha. } \mathrm{kg}^{-1}\end{array}$ & $\begin{array}{c}b \\
\text { ha. } \mathrm{kg}^{-1}\end{array}$ & $c$ & $\begin{array}{l}d \\
-\end{array}$ & $\begin{array}{c}\text { FSTR } \\
\mathrm{kg} \cdot \mathrm{kg}^{-1}\end{array}$ & $\begin{array}{c}R G R L \\
\left({ }^{\circ} \mathrm{C} \cdot \mathrm{dia}\right)^{-1}\end{array}$ \\
\hline Valores & 0,0019 & 0,0005 & $-4,5^{* *}$ & $0,14^{* *}$ & 0,171 & $0,02^{*}$ \\
\hline
\end{tabular}
crescimento relativo da área foliar $\left(R G R L,\left({ }^{\circ} \mathrm{C} . \mathrm{dia}\right)^{-1}\right)$

* valor obtido através de ajuste no modelo em função dos dados observados no campo

** valores da variedade cultivada padrão (IR-72) estabelecidos no modelo 


\section{RESULTADOS E DISCUSSÃO}

\section{Determinação da sensibilidade fotoperió- dica da variedade cultivada de ter-ras altas BRS-Primavera}

A sensibilidade fotoperiódica da variedade cultivada BRS-Primavera foi quantificada pelo valor do coeficiente angular de uma regressão linear estabelecida entre o número de dias compreendidos entre a emergência e a iniciação da panícula em função de suas respectivas datas de semeadura. De acordo com Fukai (1999), quanto maior o coeficiente angular maior é a sensibilidade fotoperiódica da variedade cultivada. Fukai (1999) dividiu a sensibilidade fotoperiódica em função dos coeficientes angulares em quatro níveis: (nível 1) insensível $(0-0,3)$, (nível 2) mediamente sensível $(0,3-0,7)$, (nível 3) altamente sensível $(0,7-0,8)$ e (nível 4) extremamente sensível $(0,8-0,9)$. $O$ valor do coeficiente angular da regressão obtido para a variedade cultivada BRS-Primavera foi de 0,0115, caracterizando sua insensibilidade fotoperiódica. A caracterização fotoperiódica é observada na regressão linear da Figura 2 A.
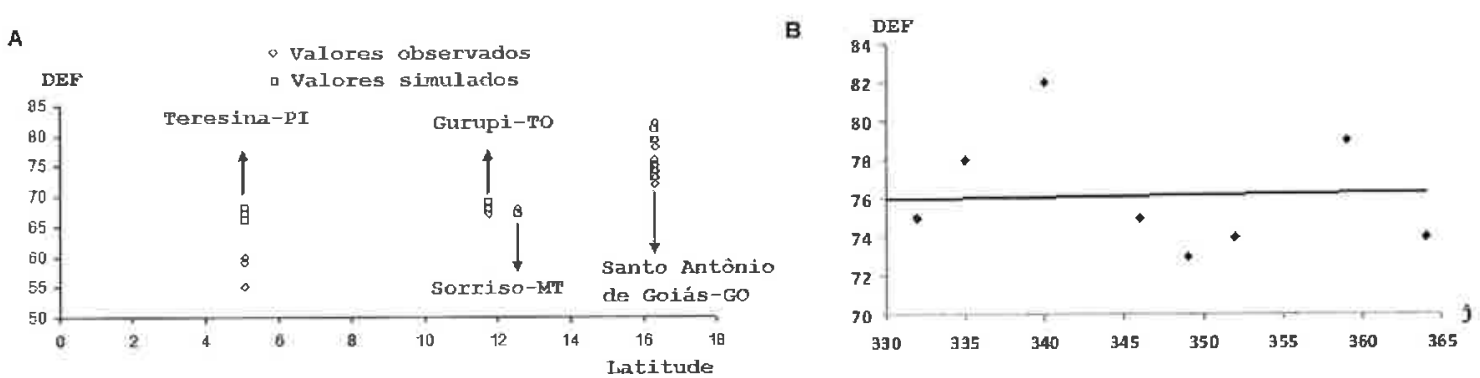

Figura 2. (A) avaliação da duração das fases 1 e 2 (emergência ao florescimento). Comparação entre os números de dias simulados e observados entre a emergência e o florescimento (DEF, dia) da variedade cultivada de terras altas BRS-Primavera em Teresina-PI, Gurupi-TO, Sorriso-MT e Santo Antônio de Goiás-GO.

\section{CONCLUSÕES}

Em função dos resultados obtidos, pode-se concluir que o modelo ORYZAAPSIM: (i) não apresentou bom desempenho em simular a duração das fases 1 e 2 (emergência ao florescimento) da variedade cultivada de arroz de terras altas BRSPrimavera (o modelo superestima - aumenta o número de dias - a duração dessa fase em regiões de menores latitudes); (ii) em Santo Antônio de Goiás-GO, apresentou bom desempenho na caracterização do crescimento da cultura na primeira data de semeadura, porém não apresentou bom desempenho na caracterização da variação temporal da massa de matéria seca total (MST, kg.ha ${ }^{-1}$ ), massa de matéria seca de folhas verdes (MSF, $\left.\mathrm{kg} \cdot \mathrm{ha}^{-1}\right) \mathrm{e}$ índice de área foliar (IAF, $\mathrm{m}^{2} \cdot \mathrm{m}^{-2}$ ) na segunda data de semeadura, pois as simulações da $M S T$ e $M S F$ e do $I A F$ foram comprometidas pelas penalizações utilizadas quando ocorre estresse hídrico e (iii) demonstrou desempenho satisfatório na simulação da produtividade do arroz de terras altas para as diferentes localidades ao nível de significância de $10 \%$ de probabilidade. 


\section{REFERÊNCIAS BIBLIOGRÁFICAS}

FUKAI, S. 1999. Phenology in rainfed lowland rice. Field Crops Research. 64: $51-60$.

GAYDON, D.S.; LISSON, S.N.; XEVI, E. 2006. Application of APSIM 'multipaddock' to estimate whole-of-farm water-use efficiency, system water balance and crop production for a rice-based operation in the Coleambally Irrigation District, NSW, Disponível em:

$<$ http://www.regional.org.au/au/asa/20 06/concurrent/water/4632 gaydond.ht $\mathrm{m}>$. Acesso em: 11 ago. 2009.

HEINEMANN, A.B.; HOOGENBOOM, G.; FARIA, R.T. 2002. Determination of spatial water requirements at county and regional levels using crop models and GIS An example for the State of Paraná, Brazil. Agricultural and Water Management. 52: 177-196.

HEINEMANN, A.B.; HOOGENBOOM, G.; GEORGIEV, G.A.; FARIA, R.T.; FRIZZONE, J.A. 2000. Center pivot irrigation management optimization of dry beans in humid areas.

Transactions of the American Society of Agricultural Engineers.6:
1507-1516.

HOOGENBOOM, G.; WILKENS, P.W.; TSUJI, G.Y. 1999. DSSAT Version 3. Honolulu: University of Hawaii. 4: 236.

JONES, J.W.; HOOGENBOOM, G.; PORTER, C.H.; BOOTE, K.J.; BATCHELOR, W.D.; HUNT, L.A.; WILKENS, P.W.; SINGH, U.; GIJSMAN, A.J.; RITCHIE, J.L. The DSSAT cropping system model. 2003. European Journal of Agronomy. 18: 235-265.

KROPFF, M.J.; VAN LAAR, H.H.; MATTHEWS, R.B. ORYZA-1: An ecophysiological model for irrigated rice production. In: SARP RESEARCH, 1994. Los Baños.

Proceedings... Wageningen:

International Rice Research Institute, 1994. 110p.

ZHANG, X.; LEE, J.H.C.; ABAWI, Y.; KIM, Y.; McCLYMONT, D.; KIM, H.D. 2007. Testing the simulation capability of APSIM-ORYZA under different levels of nitrogen fertilizer and transplanting time regimes in Korea. Australian Journal of Experimental Agriculture. 47: 14461454. 